\title{
Establishment of an osteoporosis model in tree shrews by bilateral ovariectomy and comprehensive evaluation
}

\author{
YAOLONG WANG $^{1 *}$, ZHAOXIA MA $^{1 *}$, YUANYUAN ZHENG ${ }^{2 *}$, BAOLING LIU $^{2 *}$, PENGFEI BAO $^{1}$, \\ XINGFEI WU ${ }^{1}$, CONGTAO YU ${ }^{1}$, ZHENGQI WEN ${ }^{2}$, TIEKUN MA ${ }^{2}$, JINXUE LIU ${ }^{1}$, CHANGE LIU ${ }^{1}$, \\ DAIPING MA ${ }^{1}$, HAIYING WU ${ }^{2}$, JUN LI $^{2}$, YONG YUAN ${ }^{3}$, NING LU ${ }^{3}$, HONGBIN ZHAO ${ }^{4}$, \\ YANJIAO LI ${ }^{1}$, SUPING YANG ${ }^{1,3}$, RONGPING ZHANG $^{5}$, JIEJIE DAI ${ }^{6}$ and MIN HU ${ }^{1}$
}

\begin{abstract}
${ }^{1}$ Yunnan Key Laboratory for Basic Research on Bone and Joint Diseases and Yunnan Stem Cell Translational Research Center, Kunming University, Kunming, Yunnan 650214; ${ }^{2}$ Department of Nuclear Medicine, The First Affiliated Hospital of Kunming Medical University, Kunming, Yunnan 650032; ${ }^{3}$ Department of Orthopaedics, The Second Affiliated Hospital of Kunming Medical University, Kunming, Yunnan 650101; ${ }^{4}$ Department of Orthopaedics, First People's Hospital of Yunnan Province, Kunming, Yunnan 650011; ${ }^{5}$ Department of Pharmacy, Kunming Medical University, Kunming, Yunnan 650500; ${ }^{6}$ Center of Tree Shrew Germplasm Resources, Institute of Medical Biology, Chinese Academy of Medical Sciences and Peking Union Medical College, Kunming, Yunnan 650118, P.R. China
\end{abstract}

Received June 19, 2018; Accepted February 14, 2019

DOI: $10.3892 /$ etm.2019.7339

\begin{abstract}
Osteoporosis (OP) treatment has always been challenging for elderly menopausal females. An animal model with a closer genetic association to human OP is essential for treatment research. Given its close genetic association to primates, the tree shrew is a suitable candidate for meeting the requirements for such an animal model. In the present study, a tree shrew OP model induced by ovariectomy (OVX), was established. Evaluation by multiple analysis methods, including blood biochemical indicators, uterus coefficients, micro-computed tomography analysis, histochemical analysis and scanning electron microscopic observation indicated that OVX was an appropriate method to establish the OP model in tree shrews. In addition, the biomolecular characteristics of OVX-induced osteoporosis were also assessed by transcriptome
\end{abstract}

Correspondence to: Professor Min Hu, Yunnan Key Laboratory for Basic Research on Bone and Joint Diseases and Yunnan Stem Cell Translational Research Center, Kunming University, 2 Puxin Road, Kunming, Yunnan 650214, P.R. China

E-mail: huminynkm@163.com

Professor Jiejie Dai, Center of Tree Shrew Germplasm Resources, Institute of Medical Biology, Chinese Academy of Medical Sciences and Peking Union Medical College, 935 Jiaoling Road, Kunming, Yunnan 650118, P.R. China

E-mail: lzldjj@gmail.com

*Contributed equally

Key words: osteoporosis, ovariectomy, tree shrew, micro-computed tomography analysis, histochemical analysis, scanning electron microscopic observation, bioinformatics analysis sequencing and bioinformatics analysis. The present study provides the methods used to confirm the successful establishment of the OP model in tree shrew, and suggests that the OP model is appropriate for human OP research.

\section{Introduction}

Osteoporosis (OP), a disease in which the strength of bones decreases and the risk of fracture increases, has become a common public health problem (1). In China, there are $>80$ million cases of primary OP every year (2). Hip fracture induced by $\mathrm{OP}$ is one of the most severe problems of aging persons, particularly in women, as $10-24 \%$ women will succumb within one year following hip fracture (3). With an increasing elderly population worldwide, healthcare expenditures, particularly those used to treat $\mathrm{OP}$ and associated fractures, are growing annually (4). In order to develop better drug treatments and cell therapies for osteoporosis, it is highly desirable to establish an animal model of OP with a genetic makeup closer to humans for preclinical research (5).

Animals including rats, rabbits, sheep and dogs have been previously used to establish a variety of models for the study of OP (6). Although they have been widely used, they have their own disadvantages. For instance, rats have not truly achieved skeletal maturity at the time-point of use; rabbits have relatively less cancellous bone resulting in inconvenience for bone densitometry; sheep have been used less frequently due to the high cost and time consumption; the osteoporosis model in sheep was established by ovariectomy for $\geq 12$ months post-operatively (7). Furthermore, they are not similar to humans in terms of their living environment and social psychology, and there are significant genetic differences between rodents and humans, meaning that these models are unsatisfactory for certain diseases, such as hepatitis and AIDS (8). Non-human primates, including monkeys, are 
genetically similar to humans compared to other experimental animals, making the simulation of human pathology and physiology relatively accurate. However, their high cost, low reproducibility and ethical concerns severely limit their use as experimental models $(9,10)$.

The tree shrew (Tupaia belangeri), is widely distributed in South Asia, Southeast Asia and Southwest China (11). It is characterized by short reproductive and life cycles, high reproductivity, moderate size and easy feeding. For decades, tree shrews have been increasingly used as models of human disease as they are close relatives to primate and have many human-like characteristics (12-18). Whole-genome sequencing revealed that tree shrews have a higher homology with humans than mice, rats and dogs (19). Tree shrews have been reported as models of hepatitis virus infection, myopia, social stress and depression (20). The present group has previously reported that tree shrews are suitable to establish an osteoporosis model using bilateral ovariectomy (21). However, the model remains to be deemed suitable for the detection of OP occurrence and requires a comprehensive evaluation and analysis compared with human osteoporosis. In the present study the physical and molecular changes of tree shrews suffering from OP were examined and further compared with human patients with OP. Furthermore, a method to evaluate the OP model in tree shrews was established, which may be used to investigate the therapeutic response of OP as an experimental model.

\section{Materials and methods}

Animals, grouping and treatment. A total of 12 healthy female

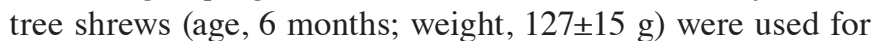
the present study. They were purchased from the Institute of Medical Biology of the Chinese Academy of Medical Sciences (Kunming, China; cat. no. SCXK-K 2013-0003), kept under standard conditions (all tree shrews were housed in the same room under a 12-h light/dark cycle with $60 \pm 10 \%$ relative humidity and temperature of $25 \pm 2^{\circ} \mathrm{C}$, with ad libitum access to water and food) for two weeks and were randomly divided into an experimental group (OVX group, $\mathrm{n}=6$ ) and a Control group (Sham group, $n=6$ ). In the present study, the tree shrews of the two groups were anesthetized by administering pentobarbital sodium via intraperitoneal injection (dose, $40 \mathrm{mg} / \mathrm{kg}$ ) (22). After anesthesia, an incision was made in the middle of the abdomen into the abdominal cavity under aseptic conditions, the bilateral ovaries were removed in the ovariectomy (OVX) group, and the same amount of greater omentum was removed in the Sham group. A diagram of the experimental design of the study is presented in Fig. 1. The animals were kept warm, and their feeding behavior and activity were closely observed and recorded. After the surgery, bone mineral density (BMD) analysis was performed every month. After 6 months, the BMD was reduced in the OVX group compared with that in the Sham group, and the tree shrews were euthanized for subsequent analysis (21). All experimental procedures were performed in accordance with the guidelines of the Kunming University Committee for Care and Use of Laboratory Animals, which followed the NIH's Guide for the Care and Use of Experimental Animals, (Kunming, China) and were approved by the Animal Experiments Ethics Committee of Kunming University (Kunming, China).
Biochemical parameter analysis of blood samples. During euthanasia, the blood was immediately extracted and centrifuged at $3,000 \times \mathrm{g}$ for $10 \mathrm{~min}$ at $4^{\circ} \mathrm{C}$ after incubation at room temperature for $2 \mathrm{~h}$. The serum was collected and stored at $-20^{\circ} \mathrm{C}$ for further biochemical analysis. According to the manufacturer's protocols (Shanghai Enzyme-linked, Shanghai, China), bone alkaline phosphatase (BALP; cat. no. ml627904), osteocalcin (BGP; cat. no. ml625695), procollagen type I N-terminal propeptide (PINP; cat. no. ml6038002), procollagen type I C-terminal propeptide (PICP; cat. no. ml6036832), cross-linked N-telopeptide of type I collagen (NTXI; cat. no. ml0281751), cross-linked N-telopeptides of type I collagen (CTXI; cat. no. m10263011), tartrate-resistant acid phosphatase (TRAP; cat. no. ml0259071), calcium (Ca; cat. no. ml058009), phosphorus (P; cat. no. ml058011) and estradiol $\left(\mathrm{E}_{2}\right.$; cat. no. ml0216351) in tree shrew serum were determined.

Determination of uterus coefficients. Prior to sacrifice, the tree shrews were weighed, and after sacrifice, the uterus of each tree shrew was completely separated and weighed. Subsequently, the uterus coefficients were calculated as follows: Uterus coefficient $=$ wet weight of the uterus/body weight.

Micro-CT scanning and analysis. Directly after euthanasia, the complete third lumbar vertebrae were collected from the tree shrews of the OVX and Sham groups. The muscles and connective tissues were peeled off and then taken analyzed by micro-computed tomography (CT) scanning (Skyscan 1272; Bruker Corp., Billerica, MA, USA). Micro-CT analysis was performed at the National \& Regional Engineering Laboratory of Tissue Engineering, Third Military Medical University (Chongqing, China).

$\mathrm{BMD}$, bone tissue volume fraction (BV/TV), bone surface/volume ratio (BS/BV), trabecular number (Tb.N), trabecular thickness (Tb.Th) and trabecular separation (Tb.Sp) were measured separately using the CT analyser.

Histological analysis. After micro-CT scanning, the third lumbar vertebrae were fixed in $4 \%$ paraformaldehyde for $72 \mathrm{~h}$, and then decalcified by soaking in $25 \%$ formic acid for 3 days. For paraffin-embedded samples, the cross section was sliced for hematoxylin-eosin (HE), ALP and TRAP staining. A HE staining kit was purchased from Beijing Solarbio Sciences \& Technology Co., Ltd (Beijing, China; cat. no. G 1120). In brief, the sections were dewaxed, washed for 2 min and stained with hematoxylin for $1 \mathrm{~min}$. Subsequently, the samples were washed with water and differentiation solution for $6 \mathrm{sec}$ at room temperature. The sections were counterstained with eosin for $1 \mathrm{~min}$ and washed with absolute ethanol, sealed with neutral gum and then examined by microscopy. ALP staining was performed with a 5-bromo-4-chloro-3'-indolyphosphate (BCIP)/(nitro-blue tetrazolium) NBT alkaline phosphatase color development kit (cat. no. C3206; Beyotime Institute of Biotechnology, Shanghai, China). In brief, the sections were dewaxed for $10 \mathrm{~min}$, washed with distilled water for $2 \mathrm{~min}$, air-dried and placed into BCIP/NBT dye working buffer containing $3 \mathrm{ml}$ color buffer, $10 \mu \mathrm{l}$ BCIP solution and $20 \mu \mathrm{l}$ NBT solution for $3 \mathrm{~h}$ at room temperature, then washed with water, air-dried, and subjected to microscopic examination. The TRAP staining kit 


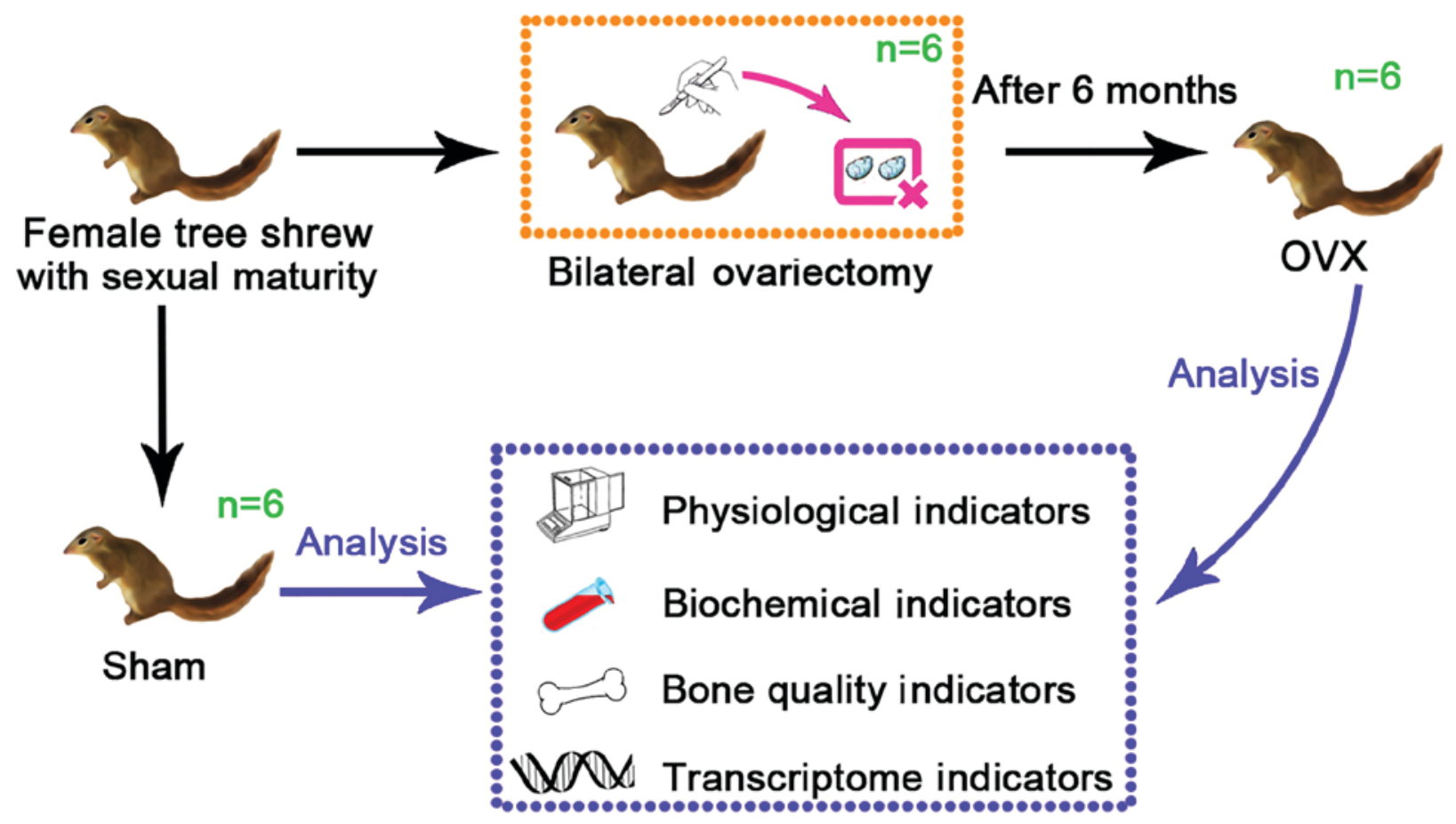

Figure 1. Diagram illustrating the establishment of the osteoporosis model in tree shrews and the analytical verification in the present study. OVX, ovariectomy.

was purchased from Beijing Solarbio Sciences \& Technology Co., Ltd (cat. no. G 1492). In brief, the sections were dewaxed for $10 \mathrm{~min}$, washed with distilled water for $2 \mathrm{~min}$, air-dried and placed into TRAP fixing solution for $1 \mathrm{~min}$. The sections were incubated in TRAP incubation buffer containing $1 \mathrm{ml}$ $\beta$-naphthol AS-BI buffer, $0.1 \mathrm{ml}$ fast garnet GBC dye solution and $9 \mathrm{ml}$ TRAP buffer for $1.5 \mathrm{~h}$ at $37^{\circ} \mathrm{C}$ and then washed with distilled water. The sections were counterstained in hematoxylin for $5 \mathrm{~min}$ at room temperature, washed, air-dried and microscopically examined. ALP and TRAP staining of lumbar spines in the sham group and OVX group $(\mathrm{n}=6)$ were visualized under a light microscope (Nikon Eclipse Ci and NIS-Element 4.30; Nikon, Tokyo, Japan) without any further histomorphometrical analysis of the number of cells and colour intensity, as the intensity of ALP and TRAP staining was obviously different between the two groups on visual inspection.

Scanning electron microscopy (SEM) observation. The tibias were cut from tree shrews of two groups and the bone tissues were fixed with $2.5 \%$ glutaraldehyde for $48 \mathrm{~h}$. Dehydration was performed with an ethanol gradient, and the samples were dried with tert-butanol, vacuum-plated and observed by SEM (S-3400N; Hitachi, Tokyo, Japan).

Calculation of ash weight coefficient. The right humerus was cut from tree shrews of two groups and dried in an oven at $110^{\circ} \mathrm{C}$ for $2 \mathrm{~h}$. Subsequently, the dry weight of the bones was determined. The dried right humerus was put in a muffle furnace at $650^{\circ} \mathrm{C}$ for $8 \mathrm{~h}$, and the ashes were then weighed. The coefficient of ash weight was determined as follows: Coefficient $=$ ash weight $/$ dry weight .

Biomechanical testing. The biomechanical properties of the left humerus were evaluated by the three-point bending flexural test method. In brief, the left humerus was placed in a biomechanical testing instrument (Instron 5565; Instron, Norwood, MA, USA). The conditions were as follows: Stride distance, $20 \mathrm{~mm}$; and loading velocity, $5 \mathrm{~mm} / \mathrm{sec}$. The data were recorded with a computer, and the maximum load, maximum displacement, structural stiffness and elastic modulus were calculated.

Transcriptome analysis. Total RNA was extracted and purified from the tissues of the first lumbar vertebra of each animal using TRIzol (Takara, Dalian, China) according to the manufacturer's protocols. Total RNA was then quantified using the BioSpec-nano nucleic acid-protein quantitative instrument (Shimadzu, Tokyo, Japan). The RNA samples from the Sham and OVX groups were packed in dry ice and sent to Novogene Bioinformatics Technology Co. Ltd (Beijing, China) for further library preparation using the Agilent Bioanalyzer 2100 system (Agilent Technologies, Inc., Santa Clara, CA, USA). Library preparations were then sequenced on an Illumina Hiseq platform (Illumina, Inc., San Diego, CA, USA) and 125/150 bp paired-end reads were generated.

Specifically, DESeq R software package (1.18.0) was used to analyse the differential expression between the two groups (three biological replicates in each group). DESeq provides a model based on the negative binomial distribution to determine statistical routines for differential expression in digital gene expression data. Benjamini and Hochberg methods were used to control the error discovery rate and adjust the resulting P-value. Genes with a P-value $<0.05$ as determined by DESeq were regarded as differentially expressed. The Gene Ontology (GO) seq R package was used for enrichment analysis of differentially expressed genes and correction of gene length deviation. After correction, $\mathrm{GO}$ with $\mathrm{P}<0.05$ was considered to be significantly enriched with differentially expressed genes. 
The database of Kyoto Encyclopedia of Genes and Genomes (KEGG; http://www.genome.jp/kegg/) was used in bioinformatics analysis. KOBAS 3.0 software (http://kobas.cbi.pku. edu.cn/) was used to test the statistical enrichment of differentially expressed genes in KEGG pathways. Next, the gene expression in healthy vs. OP patients was compared with that of tree shrews in the Sham vs. OVX group. The bone tissue samples were obtained from iliac bone in humans without OP and femoral neck in patients with OP from January 2016 to December 2018 from the first affiliated hospital of Kunming Medical University (Kunming, China). As for the tree shrew samples, RNAs from the bone tissues of humans were extracted and subjected to sequencing analysis. The sequencing results of bone tissues from healthy individuals and OS patients were compared with those of tree shrews, and genes associated with bone formation were selected for analysis. Based on the RNA-seq analysis, count per million reads was used to denote the gene expression levels.

Statistical analysis. Values are expressed as the mean \pm standard deviation and analyzed using SPSS 17.0 software for Windows (SPSS, Inc., Chicago, IL, USA). The statistical differences among groups were analyzed by using the Student's t-test. $\mathrm{P}<0.05$ was considered to indicate a statistically significant difference.

\section{Results}

Blood biochemical indicator analysis indicates high bone turnover and bone loss rate after OVX. In order to detect the changes of blood biochemical indicators in tree shrews, the levels of $\mathrm{E}_{2}$, Ca, P, BALP, BGP, PINP, PICP, NTXI, CTXI and TRAP in serum samples from the two groups were determined using the appropriate assay kits (Fig. 2). The levels of serum $E_{2}$ in the OVX group were significantly lower than those in the Sham group $(\mathrm{P}<0.001$; Fig. 2A). The Sham group appeared to have decreased $\mathrm{Ca}$ and $\mathrm{P}$ levels compared with the OVX group, but the changes were not significant ( $\mathrm{P}>0.05$; Fig. $2 \mathrm{~B}$ and $\mathrm{C}$ ). In addition, the serum biomarkers of bone formation (BALP, BGP, PINP and PICP) and bone resorption (NTXI, CTXI and TRAP) were evaluated. These parameters were significantly increased in the OVX group compared with those in the Sham group $(\mathrm{P}<0.01$; Fig. 2D-J). These results demonstrated that there was a significant difference between the Sham group and the OVX group in certain aspects of blood biochemical indicators, and the increase of bone metabolism markers reflects the high bone turnover and bone loss rate in OVX group. The bilateral OVX significantly reduced the $\mathrm{E}_{2}$ levels.

OVX reduces the uterus coefficient in tree shrews. In order to calculate the uterus coefficients in the Sham group and OVX group, the body weight and uterus weight of tree shrews from the two groups were determined. As presented in Fig. 3, the body weight in the OVX group was significantly increased throughout the treatment period compared with that in the Sham group $(\mathrm{P}<0.001$; Fig. 3A), despite the same amount of food provided to all animals. The OVX group appeared to have a significantly decreased uterus weight compared with that in the Sham group $(\mathrm{P}<0.05$; Fig. $3 \mathrm{~B})$. The uterus coefficient in the OVX group was significantly decreased compared with that in the Sham group $(\mathrm{P}<0.01$; Fig. $3 \mathrm{C})$. The above results suggested that bilateral OVX significantly reduces the uterus coefficient.

Micro-CT and histochemical analysis. The results of the micro-CT imaging revealed that the number and connections of trabeculae in the third lumbar vertebra of OVX tree shrews were obviously decreased compared with the sham group (Fig. 4A). The results of the HE staining indicated that the trabecular bone was compact, intact and continuous in the Sham group compared with that in the OVX group (Fig. 4B). The results of the microarchitectural analysis by micro-CT were then quantified for statistical comparison between the groups. The BMD of the third lumbar vertebra from the OVX group was significantly decreased compared with that in the Sham group $(\mathrm{P}<0.01$; Fig. $4 \mathrm{C})$. The $\mathrm{BS} / \mathrm{BV}$ in the $\mathrm{OVX}$ group was significantly increased compared with that in the Sham group $(\mathrm{P}<0.05$; Fig. 4D). Furthermore, the BV/TV and the Tb.Th in the OVX group were significantly decreased compared with those in the Sham group ( $\mathrm{P}<0.05$; Fig. $4 \mathrm{E}$ and $\mathrm{G})$. The Tb.N determined for the OVX group appeared to be decreased compared with that for the Sham group, but the difference was not significant $(\mathrm{P}>0.05$; Fig. $4 \mathrm{~F})$. In addition, the Tb.Sp in the OVX group appeared to be increased compared with that in the Sham group, but the difference was also not significant $(\mathrm{P}>0.05$; Fig. 4H). ALP and TRAP staining respectively revealed fewer osteoblasts and more osteoclasts in the OVX compared with the Sham group (Fig. 4I and J). The above results indicated the successful establishment of the experimental model.

SEM imaging, biomechanical properties and the coefficient of ash weight. In order to further determine the features of the new experimental model of OP, SEM images of the proximal epiphysis of the tibia were recorded, and the biomechanical properties of the left humerus and the coefficient of ash weight of the right humerus were determined for the Sham and OVX tree shrews (Fig. 5). SEM imaging under low magnification indicated that the meshwork structure of the trabecular bone was destroyed, while under high magnification, the collagen fibrils were regularly arranged in the OVX group. By contrast, in the Sham group, the trabecular reticular formation was observed to be structurally complete on low magnification and the collagen fibrils were regularly arranged, as identified under high magnification (Fig. 5A). The dry weight, ash weight and the coefficient of ash weight of the right humerus were determined, and it appeared that those in the OVX group were slightly decreased compared with those in the Sham group, but the differences were not significant ( $P>0.05$; Fig. 5B-D). The biomechanical properties (maximum load, maximum displacement, structural stiffness and elastic modulus formation) of the left humerus in the two groups were assessed. Compared with the Sham group, the OVX group featured a decreased maximum load, a decreased maximum displacement and decreased elastic modulus formation, and these differences were statistically significantly $(\mathrm{P}<0.05$; Fig. $5 \mathrm{E}, \mathrm{F}$ and $\mathrm{H})$. The OVX group exhibited a decreased structural stiffness compared with that in the Sham group, but no significant difference was obtained ( $P>0.05$; Fig. 5G).

Bioinformatics analysis of differentially expressed genes in the first lumbar vertebra. To explore potential 

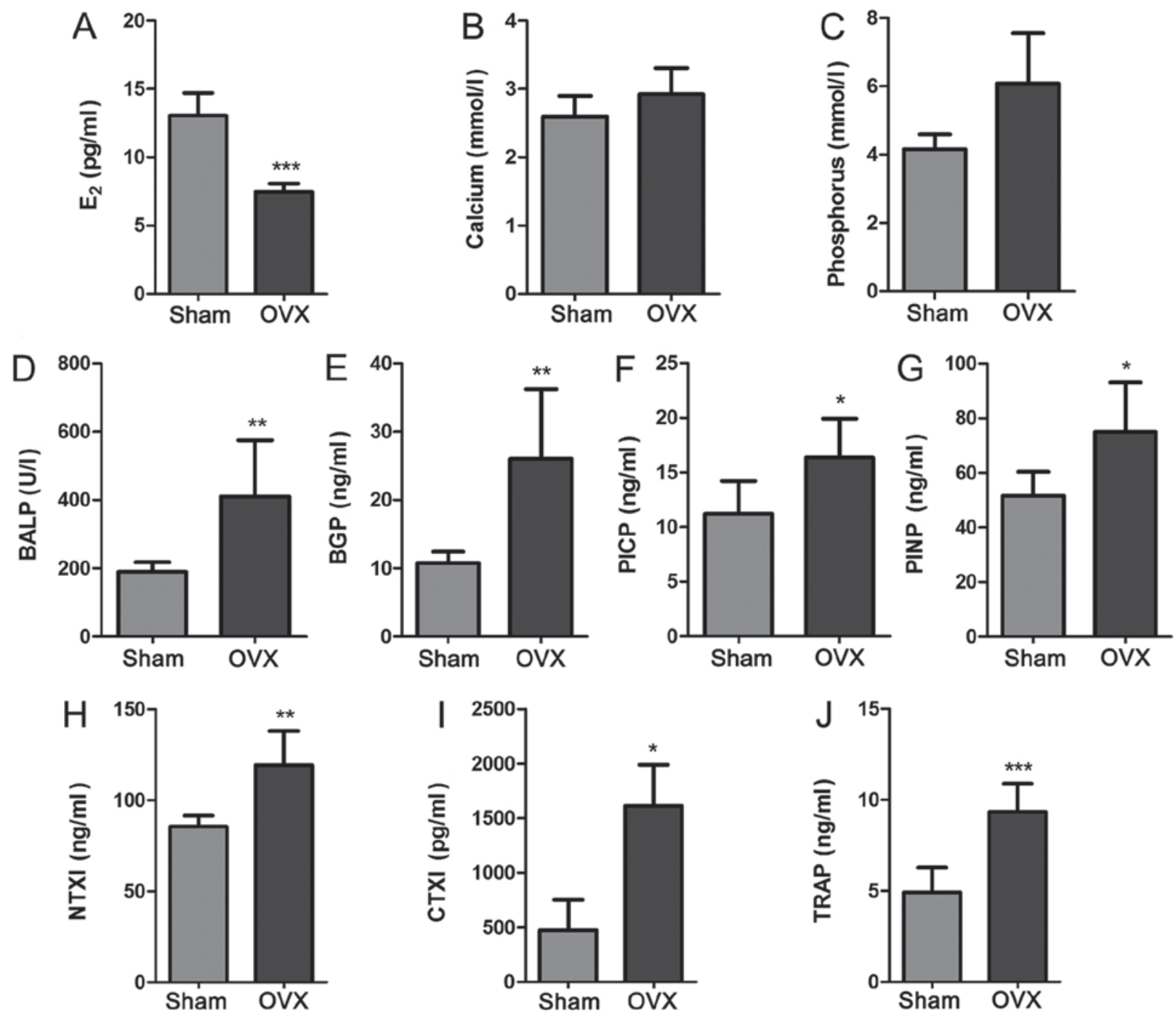

Figure 2. Blood biochemical indicators in tree shrews from the Sham and OVX groups. (A) $E_{2}$ (B) Calcium (C) Phosphorus (D) BALP (E) BGP (F) PICP (G) PINP (H) NTXI (I) CTXI (J) TRAP. Values are expressed as the mean \pm standard deviation ( $=6$ ). ${ }^{*} \mathrm{P}<0.05 ;{ }^{* * *} \mathrm{P}<0.01 ;{ }^{* * * *} \mathrm{P}<0.001$ vs. the Sham group. OVX, ovariectomy; $\mathrm{E}_{2}$, estradiol; BALP, bone alkaline phosphatase; BGP, osteocalcin; PICP, procollagen type I C-terminal propeptide; PINP, procollagen type I $\mathrm{N}$-terminal propeptide; NTXI, cross-linked N-telopeptide of type I collagen; CTXI, cross-linked N-telopeptides of type I collagen; TRAP, tartrate-resistant acid phosphatase.

underlying molecular mechanisms involved in OP development in tree shrews subjected to OVX, transcriptome analysis was performed. The results indicated that a total of 81 gene modules were identified (Fig. 6A). By using this unbiased, objective 'big-data' processing of all genes, the Fragments Per Kilobase of transcript per Million mapped reads density distribution was revealed to be different between the OVX and Sham groups (Fig. 6B). 77 gene modules were significantly differentially expressed between the two groups (Fig. 6C). Based on the 'module-trait' association plot, 33 genes were upregulated in the OVX group compared with those in the Sham group, while 44 genes were downregulated (Fig. 6C). Pathway enrichment analysis showed the upregulated genes (Table SI) in OVX group were mainly distributed in 15 pathways in which 'metabolic pathways' enriched 2 genes and the others had one gene (Fig. 7A). Among them, 'Non-homologous end-joining' had the largest enrichment factor, which was associated with DNA repair in osteoblasts (23). Among the KEGG pathways enriched by the significantly downregulated genes
(Table SII) in the OVX group, 'Drug metabolism-cytochrome P450' and 'ABC transporters' had the largest enrichment factors (Fig. 7B), all of which were associated with osteoporosis in postmenopausal woman or in aging $(24,25)$. The majority of upregulated genes were accumulated in the GO terms of 'peptidase activity', 'endopeptidase activity' and 'proteolysis' (Fig. 7C). The top $3 \mathrm{GO}$ terms for downregulated genes were 'transferase activity', 'ATPase activity' and 'single-organism biosynthetic process' (Fig. 7D) in the OP model of tree shrews.

The differential expression of certain osteogenesis-associated genes in OP was then compared between human patients and the tree shrew model by analyzing their gene expression profiles. The expression of the genes bone $\gamma$-caroboxyglutamate protein (BGLAP), integrin binding sialoprotein (IBSP), osteoprotegerin and $\mathrm{Sp} 7$ transcription factor (SP7) exhibited a downward trend without statistically significance between healthy and OP individuals in humans and tree shrews. Alkaline phosphatase, biomineralization associated was significantly decreased in patients with OP, and 

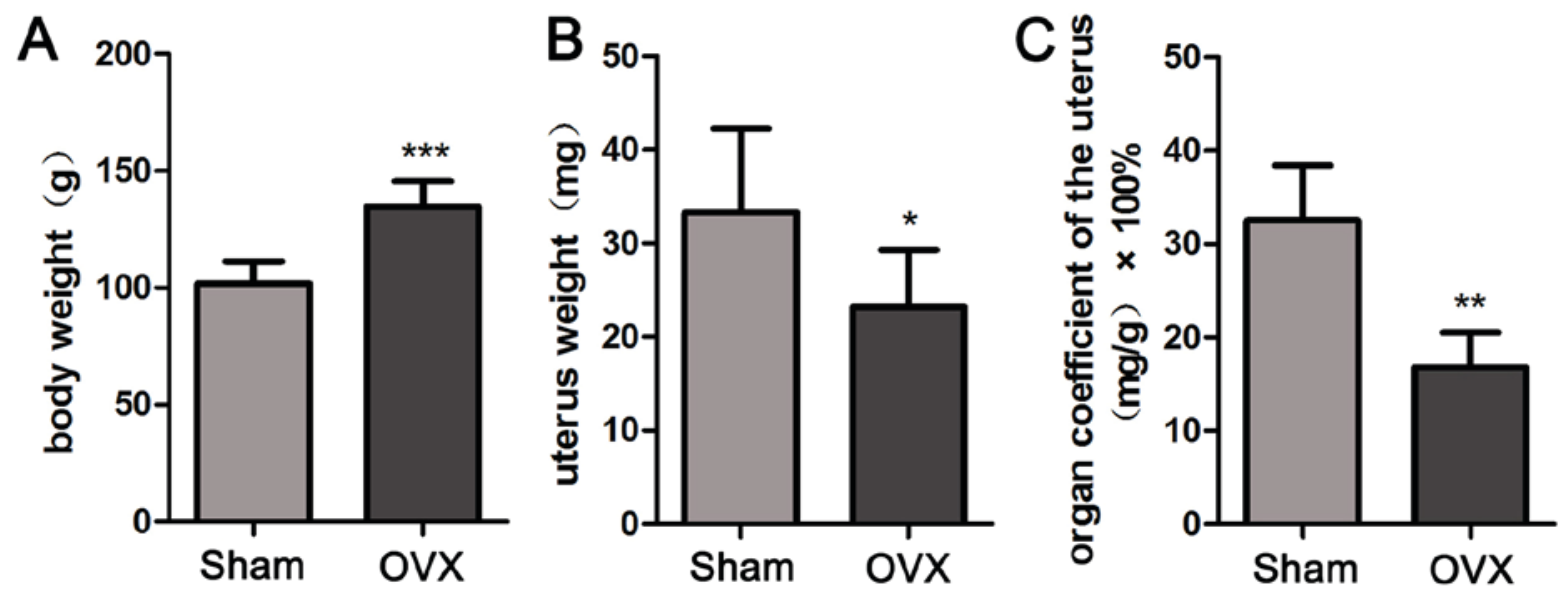

Figure 3. Uterus coefficients of tree shrews were compared between the Sham and OVX groups. (A) Body weight, (B) uterus weight and (C) organ coefficient of the uterus. Values are expressed as the mean \pm standard deviation $(\mathrm{n}=6) .{ }^{*} \mathrm{P}<0.05 ;{ }^{* *} \mathrm{P}<0.01 ;{ }^{* * *} \mathrm{P}<0.001$ vs. the Sham group. OVX, ovariectomy.

A

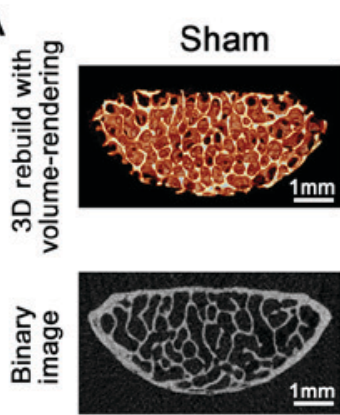

C

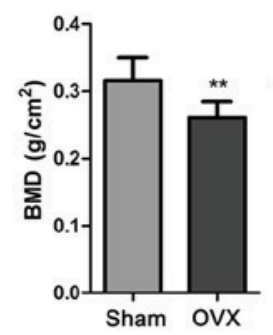

D

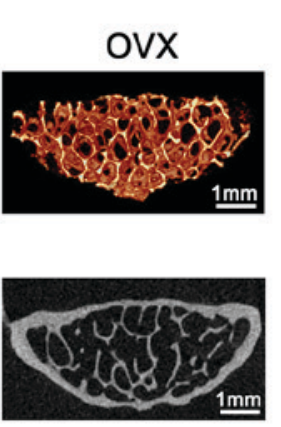

$\mathrm{E}$
B

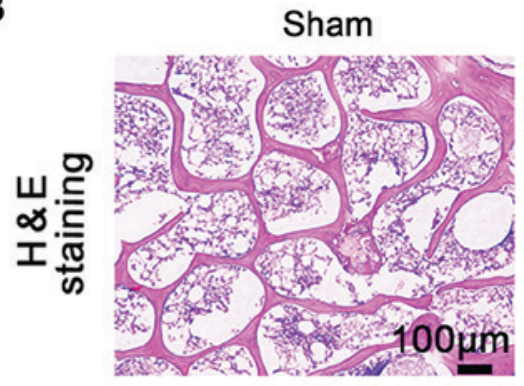

$\mathrm{F}$

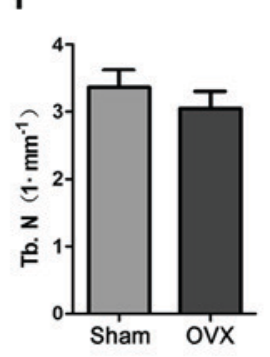

G

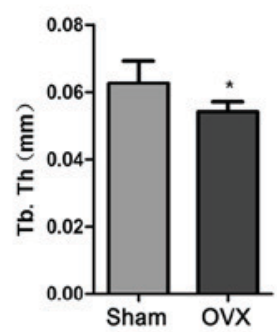

$\mathrm{H}$
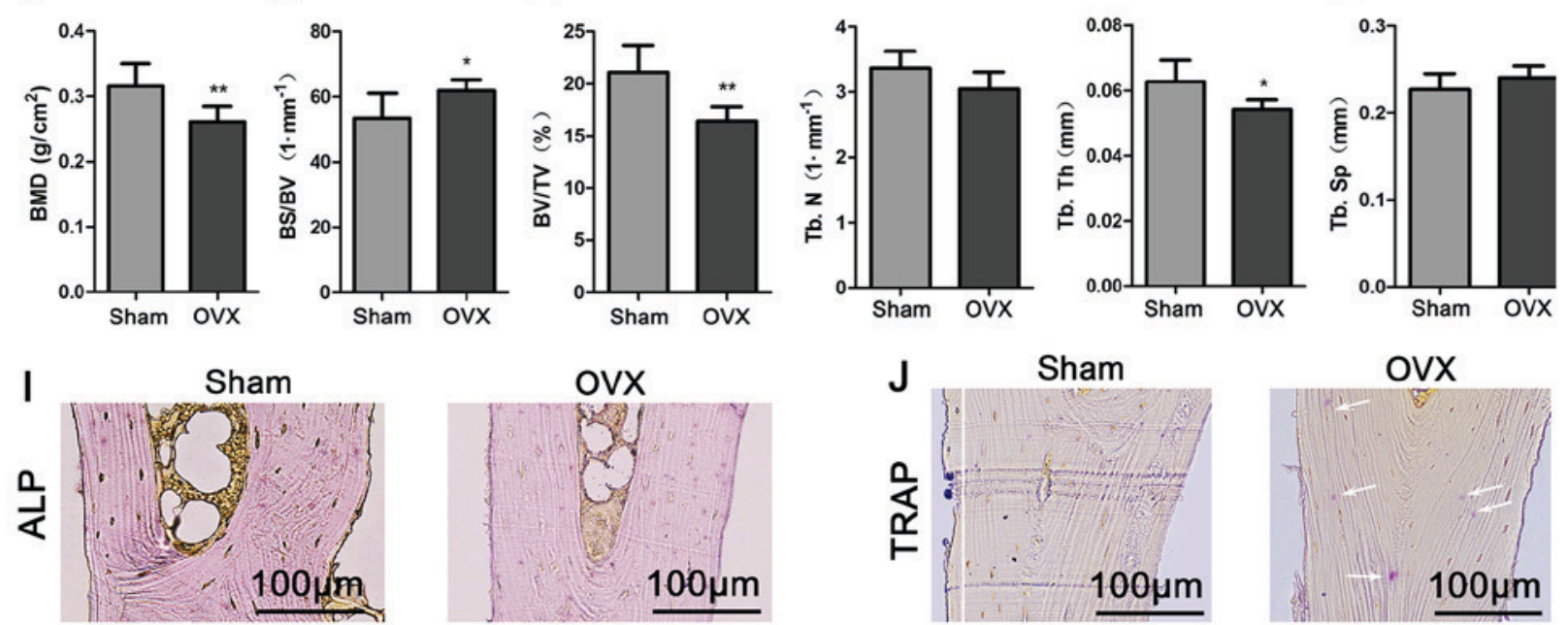

OVX

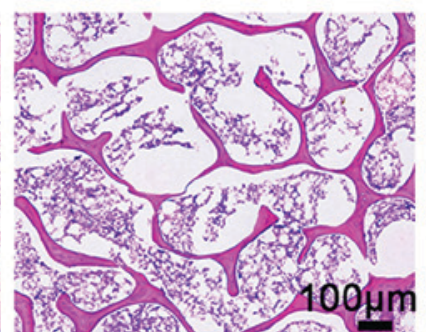

OVX

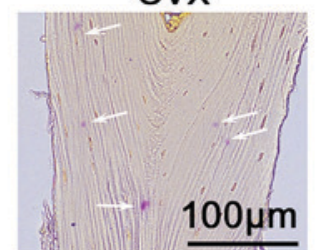

Figure 4. Third lumbar vertebra quality in the Sham and OVX groups. (A) Third lumbar vertebras were examined to micro-computed tomography imaging. (B) Third lumbar vertebras were stained with H\&E. (C-H) Evaluation of bone quality parameters of the third lumbar vertebra, including (C) the BMD, (D) BS/BV, (E) BV/TV, (F) Tb.N, (G) Tb.Th and (H) Tb.Sp. Values are expressed as the mean \pm standard deviation $(\mathrm{n}=6)$. ${ }^{*} \mathrm{P}<0.05$ and ${ }^{* *} \mathrm{P}<0.01$ vs. the Sham group. (I) ALP staining revealed the osteoblasts. (J) TRAP staining was employed to visualize the osteoclast (marked by white arrows; scale bar, $100 \mu \mathrm{m}$ ). OVX, ovariectomy; 3D, 3-dimensional; TRAP, tartrate-resistant acid phosphatase; BMD, bone mineral density; BV/TV, bone tissue volume fraction; BS/BV, bone surface/volume ratio; Tb.N, trabecular number; Tb.Th, trabecular thickness; Tb.Sp, trabecular separation; ALP, alkaline phosphatase.

exhibited similar trends without significance in the tree shrew OVX group. For the gene of runt-related transcription factor 2 (RUNX2), a significantly decreased expression level was observed both in humans and tree shrews while the expression of sclerostin was not obviously changed (Fig. S1).

\section{Discussion}

As the bones of mice are small, it is difficult to appreciate the OP macroscopic features, including reduced amounts and porosity of the cancellous bone (26). Although rats are widely used to model 
A
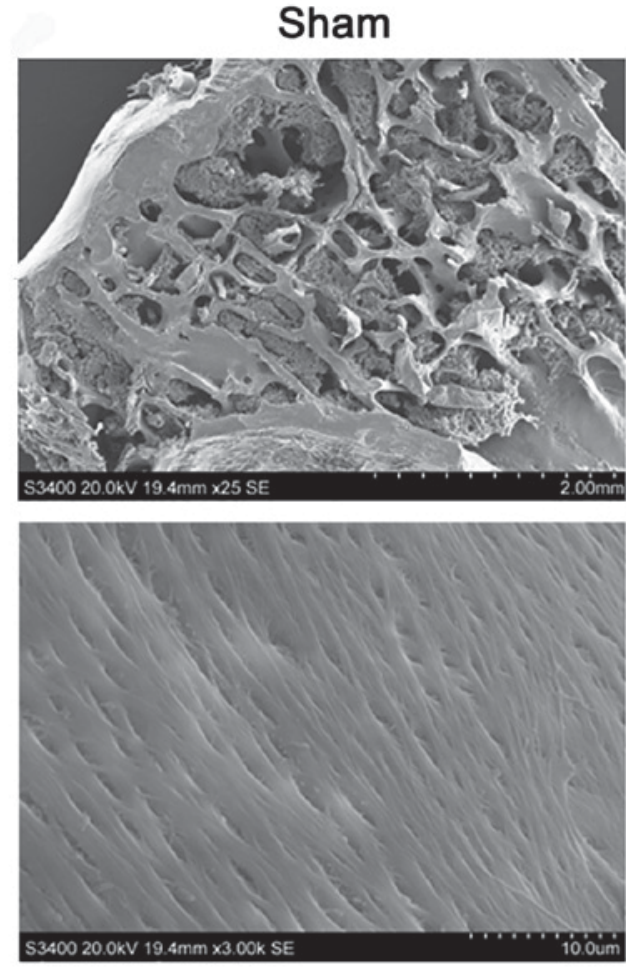

ovX
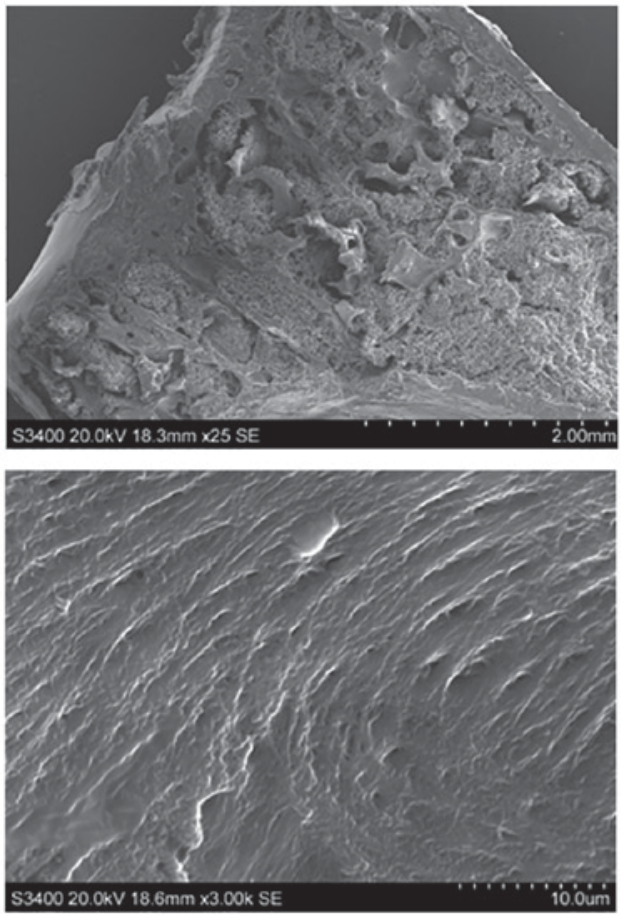
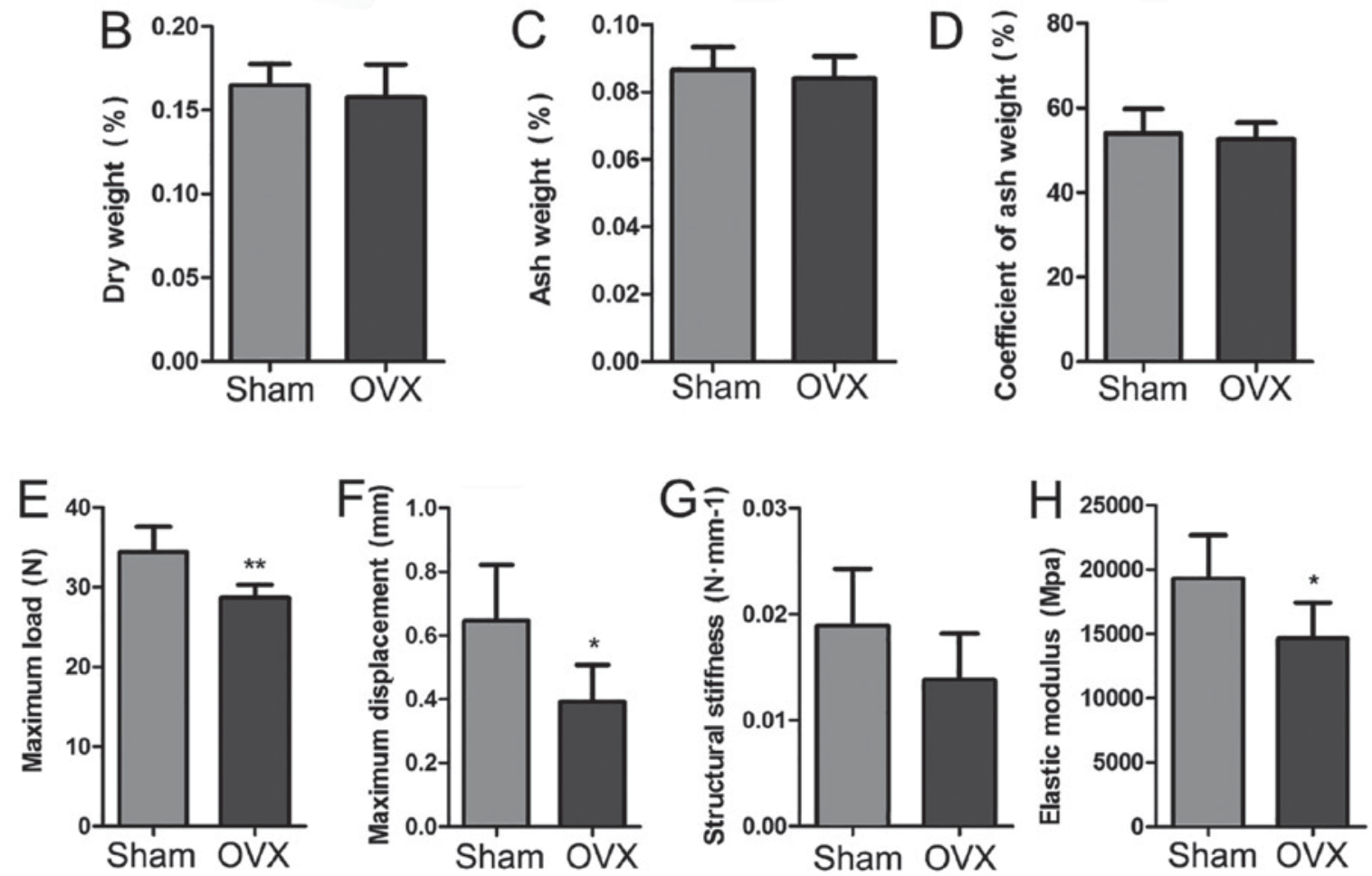

Figure 5. Quality examination of tibia and humerus in the Sham and OVX groups. (A) Representative scanning electron microscopy images of the proximal epiphysis of the tibia (scale bars: in the 25-fold magnification the scale is $2 \mathrm{~mm}$ and in the 3-thousand-fold magnification $10 \mu \mathrm{m}$ ). (B-D) Determination of the coefficients obtained from bone dust of the right humerus of tree shrews, including (B) dry weight, (C) ash weight and (D) coefficient of ash weight. (E-H) Biomechanical study of the left humerus of tree shrews from the two groups, including (E) maximum load, (F) maximum displacement, (G) Structural stiffness and $(\mathrm{H})$ elastic modulus. Values are expressed as the mean \pm standard deviation $(\mathrm{n}=6)$. " $\mathrm{P}<0.05$ and ${ }^{* * *} \mathrm{P}<0.01$ vs. the Sham group. OVX, ovariectomy.

OP, they have certain disadvantages. The major drawback of the rat skeleton is that certain bones retain lifelong growth and do not fuse epiphyses (27). The overall skeletal morphology of the tree shrew is highly similar to that of primates and the skeletal growth and bone metabolism are also much closer to humans than those of rats (28). The CT 3-dimensional visualization of tree shrews indicates that the tips of the fingers have the characteristics of claws. The length of the fingers and toes are closer to those of humans, which is suitable for grasping. At the same time, the tree shrew's brain is relatively developed, the volume of the skull cavity is large, there is a bone bridge behind the orbit and the formation of the bone orbit, the thumb and other 


\section{A}

Cluster analysis of differentially expressed genes

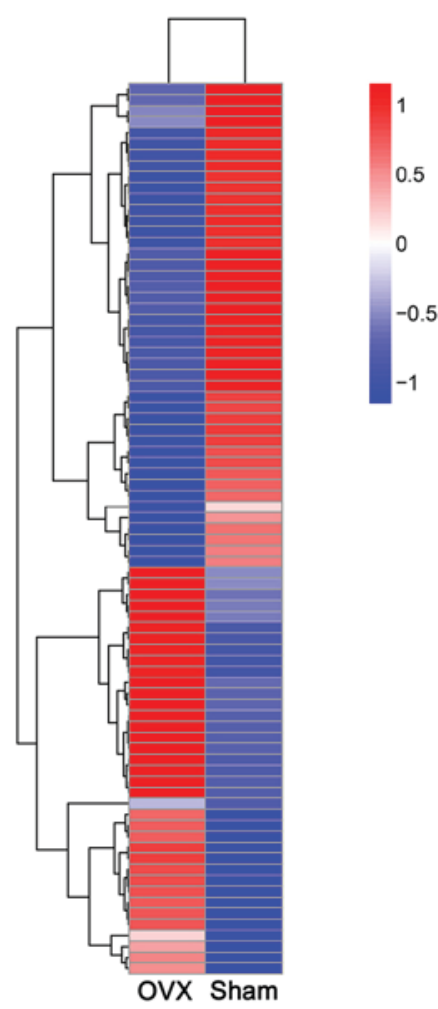

B
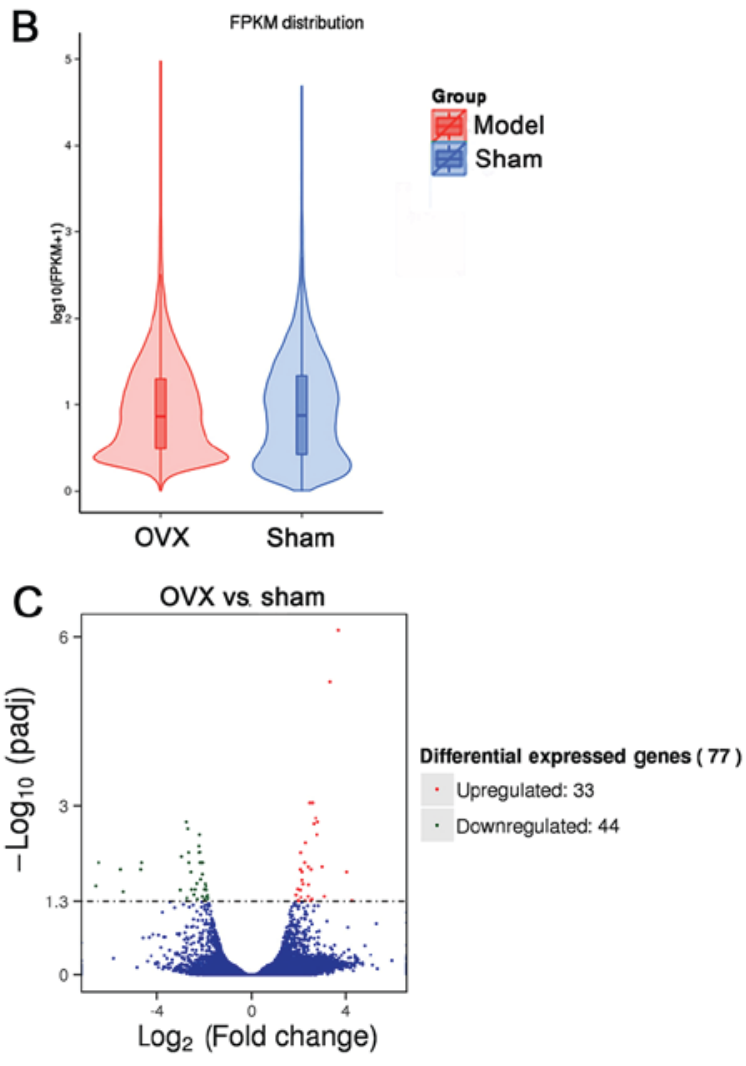

Figure 6. Bioinformatics analysis of differentially expressed genes in the first lumbar vertebra of tree shrews from the OVX vs. Sham groups. (A) Cluster analysis of differentially expressed genes (red, upregulation of gene expression; green, down-regulation of gene expression). (B) FPKM distribution. (C) Differentially expressed genes presented by fold change using a scatter diagram. OVX, ovariectomy; FPKM, fragments per kilobase of transcript per million mapped reads; padj, adjusted P-value.

fingers are separated, thereby having similar features to those of humans (29). In the present study, the tree shrew was selected as an experimental animal to induce OP and the established model was evaluated by multiple approaches.

In OP, bone loss and a high bone turnover rate are consistent; the biochemical markers of bone metabolism reflect the overall bone turnover, and may be determined to reveal the bone loss situation. The increase of bone metabolism markers reflects the high bone turnover and bone loss rate. Of note, $\mathrm{OP}$ results from an imbalance of bone resorption and bone formation. Therefore, the biochemical markers for bone formation and resorption in blood samples were investigated in the present study. As biochemical markers of bone formation, BALP, BGP, PINP, PICP may be used for detecting OP (30). BALP is a phosphatase that is elevated in OP patients with high bone turnover (31). The level of BGP, reflecting bone formation, is elevated in postmenopausal OP (32). PINP and PICP have a similar association, in terms of the occurrence of OP being accompanied by an increase in their activity (33). NTXI, CTXI and TRAP are biochemical markers of bone resorption, and may also be used for detection of OP. CTXI and NTXI are the internationally recognized biochemical markers of bone resorption, whose levels are obviously increased in patients with OP (34). The levels of TRAP reflect the activity of osteoclasts and the bone resorption status (35). In addition, the elements $\mathrm{Ca}$ and $\mathrm{P}$ are considered to be phenotypic markers of bone formation (36); in the present study, the levels of these substances were increased in the OVX group, but the changes were not significant. The menopause is closely associated with estrogen deficiency that may accelerate the pathogenesis of OP (37). In the present study, the serum levels of $E_{2}$ significantly decreased in the OVX group compared with those in the Sham group. The present study demonstrated that the changes in the plasma levels of certain biochemical markers of bone formation and resorption in OVX tree shrews were similar to those in OP patients, indicating that the OP model in tree shrews closely resembles OP in human patients, which would make it suitable for experimental studies.

In the present study, the tree shrews from the OVX group exhibited an increase in body weight compared with the Sham-operated animals, which is in agreement with the body weight changes of post-menopausal women (38). Beyond that, the uterus weight and organ coefficient of the uterus of the tree shrews in the OVX group were significantly reduced compared with those in the Sham group. This indicated that OVX tree shrews have similar physiological characteristics to post-menopausal women.

The primary objective of establishing animal models is to assess potential pharmacological and non-pharmacological approaches for the prevention and treatment of OP and associated fractures, it is desirable to establish suitable monitoring methods. As previously described, OP is a systemic skeletal disease characterized by bone loss and microstructure degradation of bone tissue, and is accompanied by increased susceptibility to bone fragility and fracture (39). BMD, biomechanical properties and bone microstructure are commonly 

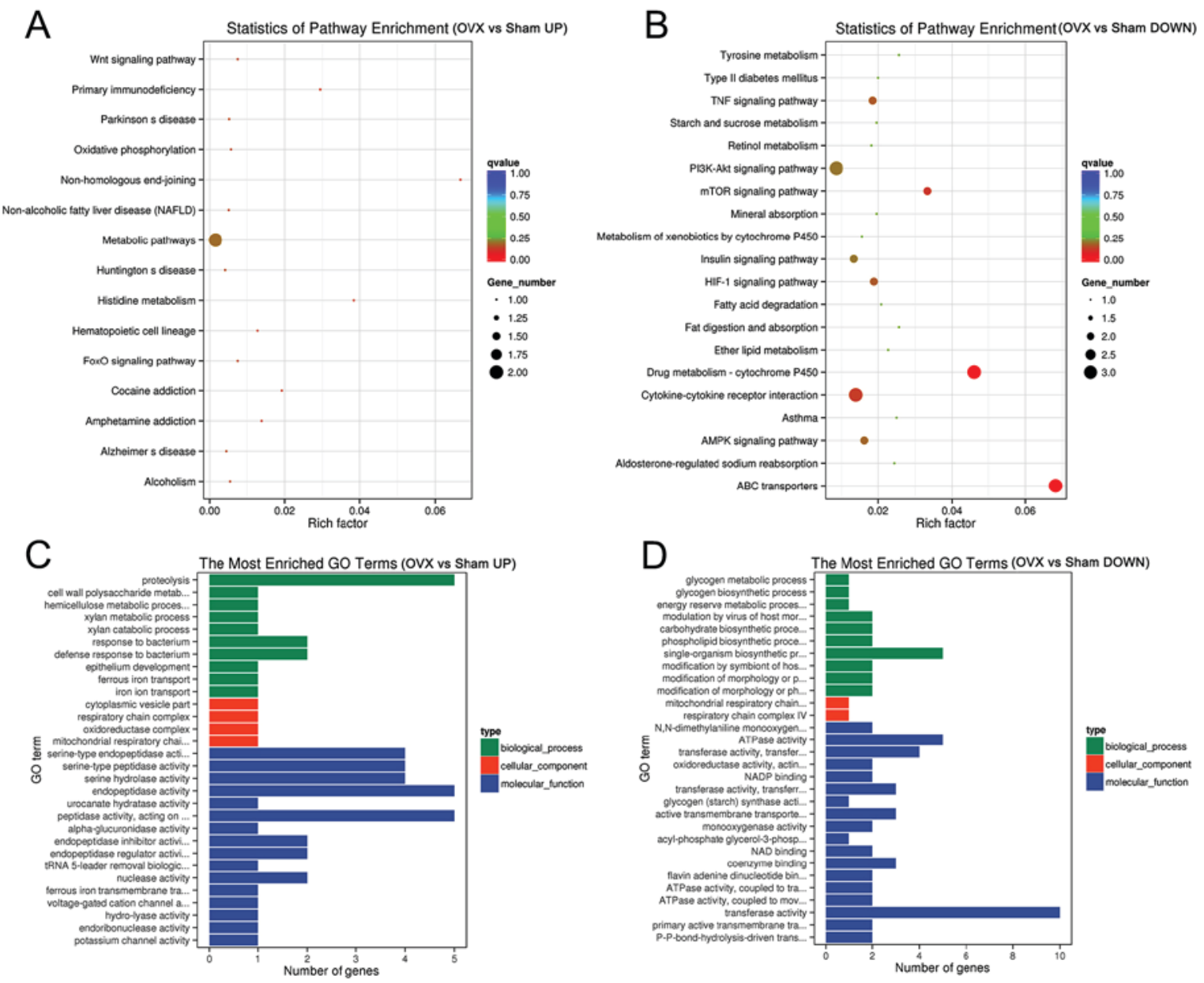

Figure 7. Bioinformatics analysis of the genetic profile of the first lumbar vertebra from the Sham and OVX groups. (A and B) Statistics of enriched pathways by (A) the upregulated and (B) the downregulated differentially expressed genes in the OVX vs. Sham group. (C and D) The most enriched GO terms (OVX vs. Sham) by (C) the upregulated and (D) the downregulated differentially expressed genes. UP, upregulated; DOWN, downregulated; GO, gene ontology; Fox, forkhead box; PI3K, phosphoinositide-3 kinase; mTOR, mammalian target of rapamycin; TNF, tumor necrosis factor; HIF, hypoxia-inducible factor; AMPK, 5' AMP-activated protein kinase; OVX, ovariectomy.

used to evaluate bone quality (40). The present results indicated that the BMD of the third lumbar vertebra in the OVX group was significantly decreased compared with that in the Sham group. The biomechanical properties (maximum deflection and maximum load) were significantly decreased in the OVX group. For the bone microarchitecture analysis, although trabecular number and trabecular separation had no significant changes, the Tb.Th and BV/TV, whose value decreased when OP occurred, were observed to be significantly decreased in the OP model in tree shrews. The BS/BV, which was negatively associated with bone mass, was markedly increased in the model. These results implied that the bone quality of tree shrews in the OVX group was significantly lower than of those in the Sham group.

In the present study, a significantly altered transcription of a large number of genes in the OVX vs. the Sham group was observed. A total of 81 differentially expressed mRNAs in the first lumbar vertebra of the Sham and OVX tree shrews were identified by RNA sequencing using the Illumina HiSeq 4000 platform. A total of $33 \mathrm{mRNAs}$ were significantly upregulated in the OVX vs. the Sham group (Table SI) and 44 mRNAs were significantly downregulated (Table SII). Among them, 5 genes named MyoD family inhibitor (MDFI), secreted frizzled-related protein 4 (SFRP4), calcyphosine like (CAPSL), oncomodulin (ONCM) and matrix extracellular phosphoglycoprotein (MEPE) are highly relevant to OP. MDFI has an important role in chondrogenesis (41). SFRP4 is involved in bone morphogenesis (42). CAPSL has a role in calcium ion binding (43). ONCM is a high-affinity calcium ion-binding protein that belongs to the superfamily of calmodulin proteins (44). MEPE regulates bone mineralization and mice lacking this gene are resistant to aging-associated trabecular bone loss (45). Genes involved in DNA repair or cell survival, including DDIAS (46), were decreased in the OVX group.

The possible biological progression, cellular functions and molecular pathways in OP were evaluated by GO and KEGG analyses on the coding genes. The results indicated that bone-associated pathways were affected in the OVX group. For instance, the WNT signaling pathway, the suppression of which may lead to OP (47), and the tumor necrosis factor signaling pathway, which is the major signaling pathway leading to bone loss (48). The upregulation of phosphoinositide-3 kinase/Akt signaling may enhance osteogenesis (49) and activation of mammalian target of rapamycin signaling pathways enhances osteogenic differentiation (50). Furthermore, the insulin signaling pathway may enhance osteogenesis (51) and 
hypoxia-inducible factor-1 signaling may promote osteoblast proliferation (52). These signaling pathways were affected in the OVX group.

In addition, the expression level of RUNX2 decreased significantly in OP patients. RUNX2 is upregulated in immature osteoblasts and is the first transcription factor required for the osteoblast differentiation (53). It regulates the expression of SP7, IBSP and BGLAP, which are associated with bone forming (54). RUNX2 was markedly changed between the Sham and OVX groups in tree shrews, which was consistent with the reduction of bone formation in OP disease.

In summary, the present study provided an experimental method to establish and evaluate an OP tree shrew model. The procedures of OVX were described in detail. The tests to evaluate the OP model in tree shrews and their results were also comprehensively outlined, and prove the successful establishment of OP in tree shrews by ovariectomy.

\section{Acknowledgements}

The authors would like to thank Mr. Qijie Dai for Micro-CT Scanning at the National \& Regional Engineering Laboratory of Tissue Engineering, The Third Military Medical University (Chongqing, China).

\section{Funding}

The present study was supported by the National Science and Key Technology Support Program (grant no. 2014BAI01B01-07).

\section{Availability of data and materials}

All data generated or analyzed during this study are included in this published article.

\section{Authors' contributions}

MH and RPZ conceived and designed the experiments; YLW, ZXM, YYZ and BLL wrote the manuscript; JJD, PFB, XFW, SPY and ZQW performed the animal experiments; JXL, CEL, DPM and TKM performed other experiments; CTY, YJL, NL and HBZ analyzed the data; HYW, JL and YY revised the manuscript. All authors read and approved the final manuscript.

\section{Ethics approval and consent to participate}

The Medical Ethics Committee of Medicine Department of Kunming University (Kunming, China) approved the use of the human tissues for scientific research purposes (permit code, FL No. 2-Ethical Review 2016). All donors provided written informed consent.

\section{Patient consent for publication}

Not applicable.

\section{Competing interests}

The authors declare that they have no competing interests.

\section{References}

1. Jackson RD and Mysiw WJ: Insights into the epidemiology of postmenopausal osteoporosis: The women's health initiative. Semin Reprod Med 32: 454-462, 2014.

2. Liu Z, Piao J, Pang L, Qing X, Nan S, Pan Z, Guo Y, Wang X, Li F, Liu J and Cheng X: The diagnostic criteria for primary osteoporosis and the incidence of osteoporosis in China. J Bone Miner Metab 20: 181-189, 2002.

3. Black DM, Arden NK, Palermo L, Pearson J and Cummings SR: Prevalent vertebral deformities predict hip fractures and new vertebral deformities but not wrist fractures. study of osteoporotic fractures research group. J Bone Miner Res 14: 821-828, 1999.

4. Dolan P and Torgerson DJ: The cost of treating osteoporotic fractures in the United Kingdom female population. Osteoporos Int 8: 611-617, 1998.

5. Graham SM, Howgate D, Anderson W, Howes C, Heliotis M, Mantalaris A, Tsiridis E and Tsapakis E: Risk of osteoporosis and fracture incidence in patients on antipsychotic medication. Expert Opin Drug Saf 10: 575-602, 2011.

6. Komori T: Animal models for osteoporosis. Eur J Pharmacol 759: 287-294, 2015.

7. Dias IR, Camassa JA, Bordelo JA, Babo PS, Viegas CA, Dourado N, Reis RL and Gomes ME: Preclinical and translational studies in small ruminants (sheep and goat) as models for osteoporosis research. Curr Osteoporos Rep 16: 182-197, 2018.

8. Glebe D and Bremer CM: The molecular virology of hepatitis B virus. Semin Liver Dis 33: 103-112, 2013.

9. Xiao J, Liu R and Chen CS: Tree shrew (Tupaia belangeri) as a novel laboratory disease animal model. Zool Res 38: 127-137, 2017.

10. Moore E: Medical relevance of UK-funded non-human primateresearch published from January 1997 to July 2012. J R Soc Med 107: 264-270, 2014.

11. Xu L, Chen SY, Nie WH, Jiang XL and Yao YG: Evaluating the phylogenetic position of Chinese tree shrew (Tupaia belangeri chinensis) based on complete mitochondrial genome: Implication for using tree shrew as an alternative experimental animal to primates in biomedical research. J Genet Genomics 39: 131-137, 2012.

12. Xu L, Zhang Y, Liang B, Lü LB, Chen CS, Chen YB, Zhou JM and Yao YG: Tree shrews under the spot light: Emerging model of human diseases. Dongwuxue Yanjiu 34: 59-69, 2013 (In Chinese).

13. Baldwin MK, Wei H, Reed JL, Bickford ME, Petry HM and Kaas JH: Cortical projections to the superior colliculus in tree shrews (Tupaia belangeri). J Comp Neurol 521: 1614-1632, 2013.

14. Petruzziello F, Fouillen L, Wadensten H, Kretz R, Andren PE, Rainer $\mathrm{G}$ and Zhang X: Extensive characterization of Tupaia belangeri neuropeptidome using an integrated mass spectrometric approach. J Proteome Res 11: 886-896, 2011.

15. Zhang XH, Dai ZX, Zhang GH, Han JB and Zheng YT: Molecular characterization, balancing selection, and genomic organization of the tree shrew (Tupaia belangeri) MHC class I gene. Gene 522: 147-155, 2013.

16. Wu X, Chang Q, Zhang Y, Zou X, Chen L, Zhang L, Lv L and Liang B: Relationships between body weight, fasting blood glucose concentration, sex and age in tree shrews (Tupaia belangeri chinensis). J Anim Physiol Anim Nutr (Berl) 7: 1179-1188, 2013.

17. Xing HJ, Jia K, He J, Shi C, Fang M, Song L, Zhang P, Zhao Y, Fu J and $\mathrm{Li}$ S: Establishment of the tree shrew as an alcohol-induced fatty liver model for the study of alcoholic liver diseases. PLoS One 10: e0128253, 2015.

18. Young PA: Genomic evidence supported tree shrew is closely related to primates. Int J Mol Evol Biodivers 3: 1-4, 2013.

19. Fan Y, Huang ZY, Cao CC, Chen CS, Chen YX, Fan DD, He J, Hou HL, Hu L, Hu XT, et al: Genome of the Chinese tree shrew. Nat Commun 4: 1426, 2013.

20. Ye LH, Meng HE, Huang YC, Zhao GQ, Lei YJ, Zhou YC and Chen XB: Tree shrew as a new animal model for the study of lung cancer. Oncol Lett 11: 2091-2095, 2016.

21. Yang F,Li YJ, Wang YL, Wang YJ,Dai JJ and Hu M:Establishment of tree shrew osteoporosis model by bilateral ovariectomy. J Kunming Medical Univ 1: 24-27, 2016 (In Chinese).

22. Zhu MC, Li H, Gyanwali B, He GY, Qi CL, Yang XM, Li ZH, Yao ZX, Wang Z and Tang A: Auditory brainstem responses after electrolytic lesions in bilateral subdivisions of the medial geniculate body of tree shrews. Neurol Sci 38: 1617-1628, 2017. 
23. Chandra A, Wang L, Young T, Zhong L, Tseng WJ, Levine MA, Cengel K, Liu XS, Zhang Y, Pignolo RJ and Qin L: Proteasome inhibitor bortezomib is a novel therapeutic agent for focal radiation-induced osteoporosis. FASEB J 32: 52-62, 2018

24. Tanaka S, Haji M, Takayanagi R, Tanaka S, Sugioka Y and Nawata H: 1,25-Dihydroxyvitamin D3 enhances the enzymatic activity and expression of the messenger ribonucleic acid for aromatase cytochrome P450 synergistically with dexamethasone depending on the vitamin D receptor level in cultured human osteoblasts. Endocrinology 37: 1860-1869, 1996.

25. Noronha-Matos JB and Correia-de-Sá P: Mesenchymal stem cells ageing: Targeting the 'purinome' to promote osteogenic differentiation and bone repair. J Cell Physiol 231: 1852-1861, 2016.

26. Harkema L, Youssef SA and de Bruin A: Pathology of mouse models of accelerated aging. Vet Pathol 53: 366-389, 2016.

27. Jee WS and Yao W: Overview: Animal models of osteopenia and osteoporosis. J Musculoskel Neuron Interact 1: 193-207, 2001.

28. Li B, Zhang RP, Li JT, He BL, Zhen H, Wang LM and Jiao JL: Measurement and analysis of anatomical parameter values in tree shrews. Dongwuxue Yanjiu 34: 132-138, 2013 (In Chinese).

29. Han YY, Xu X, Lu CX, Kuang DX, Quan PF, Wang WG, Sun XM Li N and Dai JJ: Establishment of CT 3 D visualization models and analysis of the skeletal system in adult tree shrews. Acta Lab Anim Sci Sin 25: 153-159, 2017 (In Chinese).

30. Kobayashi Y and Tokue A: Clinical usefulness of blood PICP, PINP and ICTP concentrations as bone metastasis markers in prostate cancer patients. Nihon Rinsho 56: 2072-2076, 1998 (In Japanese).

31. Haarhaus M, Monier-Faugere MC, Magnusson $\mathrm{P}$ and Malluche $\mathrm{HH}$ Bone alkaline phosphatase isoforms in hemodialysis patients with low versus non-low bone turnover: A diagnostic test study. Am J Kidney Dis 66: 99-105, 2015.

32. Zhao D, Wang J, Liu Y and Liu X: Expressions and clinical significance of serum bone Gla-protein,bone alkaline phosphatase and C-terminal telopeptide of type I collagen in bone metabolism of patients with osteoporosis. Pak J Med Sci 31: 91-94, 2015.

33. Funck-Brentano T, Biver E, Chopin F, Bouvard B, Coiffier G, Souberbielle JC, Garnero P and Roux C: Clinical utility of serum bone turnover markers in postmenopausal osteoporosis therapy monitoring: A systematic review. Semin Arthritis Rheum 41: 157-169, 2011

34. Li XP, Liu XY, Fan B and Li XY: Early changes in bone specific turnover markers during the healing process of vertebral fracture. Chin J Osteoporosis Bone Miner Res 8: 305-311, 2015 (In Chinese).

35. Azuma Y, Kaji K, Katogi R, Takeshita S and Kudo A: Tumor necrosis factor-alpha induces differentiation of and bone resorption by osteoclasts. J Biol Chem 275: 4858-4864, 2000.

36. Wang MY, Shen C, An MF, Xie CQ, Wu X, Zhu OQ, Sun B, Huang YP, Zhao YL, Wang XJ and Sheng J: Combined treatment with Dendrobium candidum and black tea extract promotes osteoprotective activity in ovariectomized estrogen deficient rats and osteoclast formation. Life Sci 220: 31-41, 2018.

37. Khosla S, Melton LJ III and Riggs BL: The unitary model for estrogen deficiency and the pathogenesis of osteoporosis: Is a revision needed? J Bone Miner Res 26: 441-451, 2011

38. Jensen LB, Vestergaard P, Hermann AP, Gram J, Eiken P, Abrahamsen B, Brot C, Kolthoff N, Sørensen $\mathrm{OH}$ Beck-Nielsen $\mathrm{H}$, et al: Hormone replacement therapy dissociates fat mass and bone mass, and tends to reduce weight gain in early postmenopausal women: A randomized controlled 5-year clinical trial of the danish osteoporosis prevention study. J Bone Miner Res 18: 333-342, 2003.

39. Kanis JA, Brazier JE, Stevenson M, Calvert NW and Lloyd JM: Treatment of established osteoporosis: A systematic review and cost-utility analysis. Health Technol Asses 6: 1-146, 2002.
40. Tang XL, Zhu TY, Hung VW, Qin L, Wong CK, Kun EW, Tam LS and Li EK: Increased organ damage associated with deterioration in volumetric bone density and bone microarchitecture in patients with systemic lupus erythematosus on longterm glucocorticoid therapy. J Rheumatol 39: 1955-1963, 2012.

41. Li J, Chen C, Bi X, Zhou C, Tao H, Ni C, Yang P, Chen S, Ye M and Duan S: DNA methylation of CMTM3, SSTR2, and MDFI genes in colorectal cancer. Gene 630: 1-7,2017.

42. Nakanishi R, Akiyama H, Kimura H, Otsuki B, Shimizu M, Tsuboyama T and Nakamura T: Osteoblast-targeted expression of Sfrp4 in mice results in low bone mass. J Bone Miner Res 23: 271-277, 2010.

43. Santiago JL, Alizadeh BZ, Martínez A, Espino L, de la Calle H, Fernández-Arquero M, Figueredo MA, de la Concha EG, Roep BO, Koeleman BP and Urcelay E: Study of the association between the CAPSL-IL7R locus and type 1 diabetes. Diabetologia 51: 1653-1658, 2008.

44. Sporeno E, Barbato G, Graziani R, Pucci P, Nitti G and Paonessa G: Production and structural characterization of amino terminally histidine tagged human oncostatin $\mathrm{M}$ in $\mathrm{E}$. coli. Cytokine 6: 255-264, 1994.

45. Nampei A, Hashimoto J, Hayashida K, Tsuboi H, Shi K, Tsuji I, Miyashita H, Yamada T, Matsukawa N, Matsumoto M, et al: Matrix extracellular phosphoglycoprotein (MEPE) is highly expressed in osteocytes in human bone. J Bone Miner Metab 22: 176-184, 2004

46. Im JY, Lee KW, Won KJ, Kim BK, Ban HS, Yoon SH, Lee YJ, Kim YJ, Song KB and Won M: DNA damage-induced apoptosis suppressor (DDIAS), a novel target of NFATc1, is associated with cisplatin resistance in lung cancer. Biochim Biophys Acta 1863: 40-49, 2016.

47. Krishnan V, Bryant HU and MacDougald OA: Regulation of bone mass by Wnt signaling. J Clin Invest 116: 1202-1209, 2006.

48. Bin G, Cuifang W, Bo Z, Jing W, Jin J, Xiaoyi T, Cong C, Yonggang C, Liping A, Jinglin M and Yayi X: Fluid shear stress inhibits TNF- $\alpha$-induced osteoblast apoptosis via ERK5 signaling pathway. Biochem Biophys Res Commun 466: 117-123, 2015.

49. Wu SS, Liang QH, Liu Y, Cui RR, Yuan LQ and Liao EY: Omentin-1 stimulates human osteoblast proliferation through PI3K/Akt signal pathway. Int J Endocrinol 2013: 368970, 2013.

50. Shen C, Kim MR, Noh JM, Kim SJ, Ka SO, Kim JH, Park BH and Park JH: Erratum to: Glucocorticoid suppresses connexin 43 expression by inhibiting the Akt/mTORsignaling pathway in osteoblasts. Calcif Tissue Int 99: 98, 2016.

51. Li BX, Wang Y, Liu Y, Ma JX and Li YK: Altered gene expression involved in insulin signaling pathway in type II diabetic osteoporosis rats model. Endocrine 43: 136-146, 2013.

52. Rankin EB, Wu C, Khatri R, Wilson TL, Andersen R, Araldi E, Rankin AL, Yuan J, Kuo CJ, Schipani E and Giaccia AJ: The HIF signaling pathway in osteoblasts directly modulates erythropoiesis through the production of EPO. Cell 149: 63-74, 2012.

53. Komori T: Regulation of osteoblast differentiation by Runx 2 . Adv Exp Med Biol 658: 43-49, 2010.

54. Komori T: Roles of Runx 2 in Skeletal Development. Adv Exp Med Biol 962: 83-93, 2017.

This work is licensed under a Creative Commons Attribution-NonCommercial-NoDerivatives 4.0 International (CC BY-NC-ND 4.0) License. 